\title{
Property of growth determined by the spectrum of operator associated to Timoshenko system with memory
}

\author{
Ronaldo Ribeiro Alves, Jaime E. Muñoz Rivera, \\ Carlos A. Raposo
}

\begin{abstract}
In this manuscript we prove the property of growth determined by spectrum of the linear operator associated with the Timoshenko system with two histories.
\end{abstract}

\section{INTRODUCTION}

This work deals with the property of growth determined by spectrum of the linear operator associated with the Timoshenko system with two histories. Timoshenko model has been considered with several beam models, we cite for instance $[5,6,11,12,15,16]$. We will use standard notation of Sobolev spaces and theory of semigroups as in $[1,7,8,9]$. Let $A$ be the infinitesimal generator of the $C_{0}$-semigroup $e^{A t}$ on a Banach space $X$. As usual, we define the type or growth order of the semigroup by

$$
w_{0}(A)=\inf \left\{w \in \mathbb{R}:\left\|e^{A t}\right\| \leq M e^{w t}, \text { for all } 0 \leq t<\infty\right\}=\inf _{t>0} \frac{\ln \left\|e^{A t}\right\|}{t}
$$

and the spectral bound by

$$
w_{\sigma}(A)=\sup \{\operatorname{Re} \lambda: \lambda \in \sigma(A)\},
$$

where $\sigma(A)$ denotes the spectrum of $A$. When $X$ has finite dimension, it is well known that

$$
w_{\sigma}(A)=w_{0}(A)
$$

In the infinite dimensional case, in general, the above equality (1.1) may not hold. From the Hille-Yosida Theorem we see that

$$
w_{\sigma}(A) \leq w_{0}(A) .
$$

2010 Mathematics Subject Classification. Primary: 35L05; Secondary: 35B40.

Key words and phrases. Timoshenko, $C_{0}$-semigroup, property of growth, memory.

Full paper. Received 16 July 2018, accepted 9 April 2019, available online 25 April 2019.

Partially supported by CAPES (Brazil). 
We notice that $w_{0}(A)$ describes the growth order of $e^{A t}$. From definition of $w_{0}(A)$ if $w>w_{0}(A)$ then there exists a constant $M \geq 1$ such that

$$
\left\|e^{A t}\right\| \leq M e^{w t}
$$

The condition (1.1) is important because it gives a practical criterion for exponential stability of $e^{A t}$. The exponential stability of $e^{A t}$ is equivalent to the condition that $w_{0}(A)<0$. If $-\infty<w_{0}(A)<0$, then the $C_{0}$-semigroup $e^{A t}$ has exponential stability. In fact, for $0<\epsilon<\left|w_{0}(A)\right|$, we have

$$
w_{0}(A)+\epsilon>\ln \left\|e^{A t}\right\| t \Rightarrow e^{\left(w_{0}(A)+\epsilon\right) t} \leq\left\|e^{A t}\right\|, \text { for all } t>N_{\epsilon} .
$$

Using the continuity of the operator $e^{A t}$ on the compact interval $\left[0, N_{\epsilon}\right]$, we obtain $M_{\epsilon}>0$ such that

$$
\left\|e^{A t}\right\| \leq M_{\epsilon} e^{\left(w_{0}(A)+\epsilon\right) t}, \text { for all } t>0,
$$

and choosing $-\mu=w_{0}(A)+\epsilon<0$, follows the exponential stability of $e^{A t}$.

In this direction the exponential stability of $e^{A t}$ is completely determined by the spectrum of $A$ and the condition (1.1) is namely of the spectrum determined growth assumption.

We use the following result duo to M. Renardy for Hilbert spaces.

Theorem 1.1. Let $H$ be a Hilbert space and $A=A_{0}+B$ the infinitesimal generator of the $C_{0}$-semigroup on $H$ where $A_{0}$ is normal and $B$ is bounded. Suppose that there exist $M>0$ and $n \in \mathbb{N}$ verifying:

(1) If $\lambda \in \sigma\left(A_{0}\right)$ and $|\lambda|>M-1$ then $\lambda$ is a isolated eigenvalue of finite multiplicity;

(2) If $|z|>M$ then that the number of eigenvalues of $A_{0}$ in the unit disk centered in $z$ (containing multiplicities) does not exceed $n$.

In this conditions we have

$$
w_{\sigma}(A)=w_{0}(A)
$$

Proof. See, [10, 13, 14].

In this work we study the Timoshenko system with two histories

$$
\begin{array}{r}
\rho_{1} \varphi_{t t}-S_{x}=0, \text { in }(0, l) \times(0, t), \\
\rho_{2} \psi_{t t}-M_{x}+S=0, \text { in }(0, l) \times(0, t), \\
S=\kappa\left(\varphi_{x}+\psi\right)-\int_{-\infty}^{t} h(t-s)\left(\varphi_{x}+\psi\right)(x, s) d s, \\
M=b \psi_{x}-\int_{-\infty}^{t} g(t-s) \psi_{x}(x, s) d s,
\end{array}
$$

with initial conditions

$$
\varphi(x, 0)=\varphi_{0}(x), \varphi_{t}(x, 0)=\varphi_{1}(x), \psi(x, 0)=\psi_{0}(x), \psi_{t}(x, 0)=\psi_{1}(x) .
$$


We consider Dirichlet-Neumann boundary condition given by

$$
\varphi(0, t)=\varphi(l, t)=\psi_{x}(0, t)=\psi_{x}(l, t)=0, t>0 .
$$

This manuscript is organized as follows. In the Section 2 we present the preliminary results, Section 3 we prove the existence of solution, Section 4 deals with the property of growth determined for spectrum and finally in the Section 5 we calculate the type of $C_{0}$-semigroup.

\section{Preliminar Results}

We denote $H$ a Hilbert space and

$$
A: D(A) \subset H \rightarrow H
$$

the infinitesimal generator of the $C_{0}$-semigroup of contractions $S(t)=e^{A t}$. Then $U(t)=S(t) U_{0}$ is solution of the equation

$$
U_{t}-A U=0, \quad U(0)=U_{0},
$$

that can be extended to

$$
U_{t t}-A U_{t}=0, \quad U(0)=U_{0}, U_{1}(0)=U_{1} .
$$

For this, is equivalent to prove that the operator

$$
\mathcal{A}_{1}=\left(\begin{array}{cc}
0 & I \\
0 & A
\end{array}\right)
$$

generates a $C_{0}$-semigroup of contractions in the space

$$
\mathcal{H}_{1}=\{(U, V) \in D(A) \times H ; A U-V \in D(A)\},
$$

provided the inner product

$$
\begin{aligned}
\left\langle\left(\begin{array}{c}
U^{1} \\
V^{1}
\end{array}\right),\left(\begin{array}{c}
U^{2} \\
V^{2}
\end{array}\right)\right\rangle_{\mathcal{H}_{1}}= & \left(V^{1}, V^{2}\right)_{H}+\left(A U^{1}-V^{1}, A U^{2}-V^{2}\right)_{H} \\
& +\left(A^{2} U^{1}-A V^{1}, A^{2} U^{2}-A V^{2}\right)_{H},
\end{aligned}
$$

with norm

$$
\left\|\left(\begin{array}{c}
U \\
V
\end{array}\right)\right\|_{\mathcal{H}_{1}}^{2}=\|V\|_{H}^{2}+\|A U-V\|_{H}^{2}+\left\|A^{2} U-A V\right\|_{H}^{2} .
$$

In the Hilbert space $\mathcal{H}_{1}$ we take the domain of $\mathcal{A}_{1}$, as

$$
D\left(\mathcal{A}_{1}\right)=\left\{\mathcal{U}=(U, V) \in \mathcal{H}_{1} ; \quad(U, V) \in D\left(A^{2}\right) \times D(A)\right\} .
$$

We have the following result.

Lemma 2.1. The operator $\mathcal{A}_{1}$ is the infinitesimal generator of a $C_{0}$-semigroup of contractions on $\mathcal{H}_{1}$. 
Proof. $D\left(\mathcal{A}_{1}\right)$ is a closed and dense space on $\mathcal{H}_{1}$. We will show that $\mathbb{R}^{+} \subset$ $\rho\left(\mathcal{A}_{1}\right)$. For $\mathcal{F}=\left(F_{1}, F_{2}\right) \in \mathcal{H}_{1}$, we will prove that there exists a unique solution $\mathcal{U} \in D\left(\mathcal{A}_{1}\right)$ such that

$$
\lambda \mathcal{U}-\mathcal{A}_{1} \mathcal{U}=\mathcal{F} .
$$

In terms of its components we get

$$
\begin{aligned}
\lambda U-V & =F_{1}, \\
\lambda V-A V & =F_{2} .
\end{aligned}
$$

As $A$ is infinitesimal generator of a $C_{0}$-semigroup of contractions, we have that for all $F_{2} \in H$ there exists a unique solution $V \in D(A)$ such that

$$
\lambda V-A V=F_{2} .
$$

Then,

$$
V=(\lambda I-A)^{-1} F_{2}
$$

and

Replacing (2.3) into (2.1) we find

$$
\left\|(\lambda I-A)^{-1}\right\| \leq \frac{1}{\lambda}
$$

$$
\lambda U-(\lambda I-A)^{-1} F_{2}=F_{1} .
$$

Applying $(\lambda I-A)$ in the above equation we have

$$
\lambda U-A U=F_{1}+\frac{1}{\lambda} F_{2}-\frac{1}{\lambda} A F_{1} \in D(A) .
$$

Since $A$ is generator infinitesimal of a $C_{0}$-semigroup, we have that $U \in$ $D\left(A^{2}\right)$. Using (2.1) and (2.2) we have

$$
\begin{aligned}
\lambda A U-A V & =A F_{1}, \\
\lambda V-A V & =F_{2} .
\end{aligned}
$$

Taking the difference of these equations

$$
\lambda(A U-V)=A F_{1}-F_{2} .
$$

Similarly,

$$
\lambda\left(A^{2} U-A V\right)=A^{2} F_{1}-A F_{2} .
$$

Getting the norms, follows

$$
\lambda\|A U-V\| \leq\left\|A F_{1}-F_{2}\right\|, \quad \lambda\left\|A^{2} U-A V\right\| \leq\left\|A^{2} F_{1}-A F_{2}\right\| .
$$

Making the inner product of (2.2) with $V$ we obtain

$$
\lambda\|V\|^{2}-\langle A V, V\rangle=\left\langle F_{2}, V\right\rangle .
$$

As $\langle A V, V\rangle \leq 0$ for all $V \in D(A)$, by Holder's inequality, follows

$$
\lambda\|V\| \leq\left\|F_{2}\right\| .
$$


From (2.4)-(2.5) we get

$$
\lambda^{2}\|\mathcal{U}\|^{2} \leq\|\mathcal{F}\|^{2}
$$

that implies

$$
\left\|(\lambda I-\mathcal{A})^{-1}\right\| \leq \frac{1}{\lambda}
$$

By Theorem of Hille-Yosida the conclusion hold.

We can extend this result to

$$
U_{t t t}-A U_{t t}=0, \quad U(0)=U_{0}, \quad U_{t}(0)=U_{1}, \quad U_{t t}(0)=U_{2} .
$$

Then, the operator

$$
\mathcal{A}_{2}=\left(\begin{array}{ccc}
0 & I & 0 \\
0 & 0 & I \\
0 & 0 & A
\end{array}\right)
$$

generates a $C_{0}$-semigroup of contractions in the space

$\mathcal{H}_{2}=\left\{(U, V, W) \in D\left(A^{2}\right) \times D(A) \times H ; A U-V \in D\left(A^{2}\right) ; A V-W \in D(A)\right\}$,

with inner product

$$
\begin{aligned}
\left\langle\left(\begin{array}{c}
U^{1} \\
V^{1} \\
W^{1}
\end{array}\right),\left(\begin{array}{c}
U^{2} \\
V^{2} \\
W^{2}
\end{array}\right)\right\rangle_{\mathcal{H}_{2}} & =\left(W^{1}, W^{2}\right)_{H} \\
& +\sum_{j=1}^{3}\left(A^{j} U^{1}-A^{j-1} V^{1}, A^{j} U^{2}-A^{j-1} V^{2}\right)_{H} \\
& +\sum_{j=1}^{2}\left(A^{j} V^{1}-A^{j-1} W^{1}, A^{j} V^{2}-A^{j-1} W^{2}\right)_{H}
\end{aligned}
$$

and domain

$$
D\left(\mathcal{A}_{2}\right)=\left\{\mathcal{U}=(U, V, W) \in \mathcal{H}_{2} ; \quad(U, V, W) \in D\left(A^{3}\right) \times D\left(A^{2}\right) \times D(A)\right\} .
$$

Now consider the following result.

Lemma 2.2. Let $A$ be the infinitesimal generator of a $C_{0}$-semigroup of contractions, then

$$
\mathcal{A}_{2}=\left(\begin{array}{ccc}
0 & I & 0 \\
0 & 0 & I \\
0 & 0 & A
\end{array}\right)
$$

verifies

$$
\rho\left(\mathcal{A}_{2}\right)=\rho(A) \cup\{0\} .
$$


Proof. Suppose $\lambda \in \rho(A)$ then exist a unique solution of the resolvent equation

$$
\lambda U-A U=F,
$$

where $F \in H$. We shall that $\lambda \in \rho\left(\mathcal{A}_{2}\right)$. Consider the problem

$$
\lambda \mathcal{U}-\mathcal{A}_{2} \mathcal{U}=\mathcal{F} .
$$

We have

$$
\begin{aligned}
\lambda U-V & =F_{1} \in D\left(A^{2}\right), \\
\lambda V-W & =F_{2} \in D(A), \\
\lambda W-A W & =F_{3} \in H .
\end{aligned}
$$

By the hypothesis there exists a unique $W \in D(A)$ solution of (2.8). So (2.7) can be written as

$$
\lambda V-(\lambda I-A)^{-1} F_{3}=F_{2},
$$

and then

$$
\lambda(\lambda I-A) V=(\lambda I-A) F_{2}+F_{3} \in D(A) .
$$

Using the hypothesis again found that there is a unique $V \in D\left(A^{2}\right)$ such that

$$
\lambda V=F_{2}+(\lambda I-A)^{-1} F_{3} \in D(A) .
$$

Substituting in (2.6) and multiplying by $\lambda$ we get

$$
\lambda^{2} U-F_{2}-(\lambda I-A)^{-1} F_{3}=\lambda F_{1} \in D\left(A^{2}\right) .
$$

From where follows

$$
\lambda^{2}(\lambda I-A) U-(\lambda I-A) F_{2}-F_{3}=\lambda(\lambda I-A) F_{1} \in D\left(A^{2}\right) .
$$

Thus

$$
\lambda^{2}(\lambda U-A U)=\lambda^{2} F_{1}+\lambda F_{2}-\lambda A F_{1}-A F_{2}+F_{3} \in D\left(A^{2}\right) .
$$

Follows that $U \in D\left(A^{3}\right)$ and show $\lambda \in \rho\left(\mathcal{A}_{2}\right)$. Reciprocally, take $\lambda \in$ $\rho\left(\mathcal{A}_{2}\right)$, then we have for all $\mathcal{F} \in \mathcal{H}_{2}$ that there exists a unique $\mathcal{U} \in D(\mathcal{A})$ verifying (2.6)-(2.8). In particular, from (2.8) we have that for all $F_{3} \in H$ there exists a unique $W \in D(A)$. So we concludes that $\lambda \in \rho(A)$.

As a consequence of the previous lemmas we have the following theorem

Theorem 2.1. Suppose that $A$ is a normal operator for which there exist $M>0$ and an integer $n$ verifying:

(1) If $\lambda \in \sigma\left(A_{0}\right)$ and $|\lambda|>M-1$, then $\lambda$ is an isolated eigenvalue of finite multiplicity, and

(2) If $|z|>M$, then the number of eigenvalues $A_{0}$ into of unit disc centered in z (containing multiplicities) does not exceed $n$. 
With this conditions the operator $\mathcal{A}$ defined by

$$
\mathcal{A}=\left(\begin{array}{lll}
0 & I & 0 \\
0 & 0 & I \\
0 & 0 & A
\end{array}\right)
$$

is a normal operator which also satisfies the properties (1) and (2).

Proof. As $A$ is self-adjoint an normal, we have that $\mathcal{A}^{*}=\mathcal{A}$, and in particular $\mathcal{A}$ is normal. Moreover, the spectra of $A$ and $\mathcal{A}$ are equal out of a unit ball and the result follows.

\section{Existence And Uniqueness of SOlUtion}

In this section, we show the existence, regularity and uniqueness of solution to the problem (1.2)-(1.6). We remember that, Dafermos [2, 3], Fabrizio [4], introduced the memory spaces by the new variables $\eta$ and $\nu$,

$$
\eta(x, t, s)=\psi(x, t)-\psi(x, t-s), \quad \nu(x, t, s)=\varphi(x, t)-\varphi(x, t-s),
$$

that satisfies

$$
\begin{array}{ll}
\eta_{t}(x, t, s)=\psi_{t}(x, t)-\psi_{t}(x, t-s), & \eta_{s}(x, t, s)=\psi_{t}(x, t-s), \\
\nu_{t}(x, t, s)=\varphi_{t}(x, t)-\varphi_{t}(x, t-s), & \nu_{s}(x, t, s)=\varphi_{t}(x, t-s) .
\end{array}
$$

Summing we find the equations

$$
\eta_{t}+\eta_{s}=\psi_{t}(x, t), \nu_{t}+\nu_{s}=\varphi_{t}(x, t) .
$$

From definitions of $\eta$ and $\nu$, we obtain the news boundary conditions

$$
\eta(x, t, 0)=0=\nu(x, t, 0), \text { for all } t \geq 0, x \in(0, l) .
$$

Moreover, follows that

$$
\begin{aligned}
& \eta(x, 0, s)=\psi(x, 0)-\psi(x,-s)=\psi_{0}(x)-\psi_{h}(x,-s):=\eta_{0}(x, s), \\
& \nu(x, 0, s)=\varphi(x, 0)-\varphi(x,-s)=\varphi_{0}(x)-\varphi_{h}(x,-s):=\nu_{0}(x, s) .
\end{aligned}
$$

We denote by $L_{\mu}^{2}\left(0, \infty ; H_{0}^{1}(0, l)\right)$ the space of square integrable functions with weight $\mu$ and values into $H_{0}^{1}(0, l)$, by

$$
L_{\mu}^{2}\left(0, \infty ; H_{0}^{1}(0, l)\right)=\left\{f \in H_{0}^{1}(0, l) ; \int_{0}^{+\infty} \mu(s) \int_{0}^{l}\left|f_{x}(x, s)\right|^{2} d x d s<\infty\right\} .
$$

This space with the inner product

$$
(f, g)_{L_{\mu}^{2}}=\int_{0}^{+\infty} \mu(s) \int_{0}^{l} f_{x}(x, s) g_{x}(x, s) d x d s
$$

is a Hilbert space.

Making change of variables and using the equation, we found

$$
\int_{-\infty}^{t} g(t-\tau) \psi_{x x}(x, \tau) d \tau=\int_{0}^{\infty} g(\tau) \psi_{x x}(x, t-\tau) d \tau
$$




$$
=-\int_{0}^{\infty} g(\tau) \eta_{x x}(x, \tau) d \tau+\int_{0}^{\infty} g(\tau) d \tau \psi_{x x}
$$

and

$$
\begin{aligned}
\int_{-\infty}^{t} h(t-\tau)\left(\varphi_{x}+\psi\right)_{x}(x, \tau) d \tau= & \int_{0}^{\infty} h(\tau)\left(\varphi_{x}+\psi\right)_{x}(x, t-\tau) d \tau \\
= & -\int_{0}^{\infty} h(\tau)\left(\nu_{x}+\eta\right)_{x}(x, \tau) d \tau \\
& +\int_{0}^{\infty} h(\tau) d \tau\left(\varphi_{x}+\psi\right)_{x}
\end{aligned}
$$

From similar way

$$
\begin{aligned}
\int_{-\infty}^{t} h(t-\tau)\left(\varphi_{x}+\psi\right)(x, \tau) d \tau= & -\int_{0}^{\infty} h(\tau)\left(\nu_{x}+\eta\right)(x, \tau) d \tau \\
& +\int_{0}^{\infty} h(\tau) d \tau\left(\varphi_{x}+\psi\right)
\end{aligned}
$$

The system (1.2)-(1.3) can be rewritten as

$$
\begin{gathered}
\rho_{1} \varphi_{t t}-\underbrace{\left(\kappa-\int_{0}^{\infty} h(\tau) d \tau\right)}_{:=\kappa_{0}}\left(\varphi_{x}+\psi\right)_{x}-\int_{0}^{\infty} h(\tau)\left(\nu_{x}+\eta\right)_{x}(x, \tau) d \tau=0, \\
\rho_{2} \psi_{t t}-\underbrace{\left(b-\int_{0}^{\infty} g(\tau) d \tau\right)}_{:=b_{0}} \psi_{x x}+\underbrace{\left(\kappa-\int_{0}^{\infty} h(\tau) d \tau\right)}_{:=\kappa_{0}}\left(\varphi_{x}+\psi\right) \\
-\int_{0}^{\infty} g(\tau) \eta_{x x}(x, \tau) d \tau+\int_{0}^{\infty} h(\tau)\left(\nu_{x}+\eta\right)(x, \tau) d \tau=0 .
\end{gathered}
$$

Then, the system (1.2)-(1.6) can be seen as

$$
\begin{aligned}
\rho_{1} \varphi_{t t}-S_{x} & =0, \text { in }(0, l) \times(0, t), \\
\rho_{2} \psi_{t t}-M_{x}+S & =0, \text { in }(0, l) \times(0, t), \\
\nu_{t}+\nu_{s} & =\varphi_{t}, \text { in }(0, l) \times(0, t), \\
\eta_{t}+\eta_{s} & =\psi_{t}, \text { in }(0, l) \times(0, t),
\end{aligned}
$$

where

$$
S=\kappa_{0}\left(\varphi_{x}+\psi\right)+\int_{0}^{\infty} h(s)\left(\nu_{x}+\eta\right)(x, s) d s
$$

and

$$
M=b_{0} \psi_{x}+\int_{0}^{\infty} g(s) \eta_{x}(x, s) d s
$$


In this way, we have the initial conditions

$$
\begin{aligned}
\varphi(x, 0) & =\varphi_{0}(x), & \varphi_{t}(x, 0) & =\varphi_{1}(x), \\
\psi(x, 0) & =\psi_{0}(x), & \psi_{t}(x, 0) & =\psi_{1}(x), \\
\nu(x, 0, s) & =\nu_{0}(x, s), & \eta(x, 0, s) & =\eta_{0}(x, s),
\end{aligned}
$$

and boundary conditions

$$
\begin{gathered}
\varphi(0, t)=\varphi(l, t)=\psi_{x}(0, t)=\psi_{x}(l, t)=0, \\
\nu(0, t, 0)=\nu(l, t, 0)=\eta(0, t, 0)=\eta(l, t, 0)=0 .
\end{gathered}
$$

Consider $\Phi=\varphi_{t}$ and $\Psi=\psi_{t}$. Let $U=(\varphi, \Phi, \nu, \psi, \Psi, \eta)^{T}$ be a vector, so we have the model (3.1)-(3.6), can be written as $U_{t}=\mathcal{A} U$ with

$$
\mathcal{A} U=\left[\begin{array}{c}
\Phi \\
\frac{\kappa_{0}}{\rho_{1}}\left(\varphi_{x}+\psi\right)_{x}+\frac{1}{\rho_{1}} \int_{0}^{\infty} h(s)\left(\nu_{x}+\eta\right)_{x}(s) d s \\
\Phi-\nu_{s} \\
\Psi \\
\frac{b_{0}}{\rho_{2}} \psi_{x x}-\frac{\kappa_{0}}{\rho_{2}}\left(\varphi_{x}+\psi\right)+\frac{1}{\rho_{2}} \int_{0}^{\infty} g(s) \eta_{x x}(s) d s-\frac{1}{\rho_{2}} \int_{0}^{\infty} h(s)\left(\nu_{x}+\eta\right)(s) d s \\
\Psi-\eta_{s}
\end{array}\right],
$$

where $U_{0}=\left(\varphi_{0}, \varphi_{1}, \nu_{0}, \psi_{0}, \psi_{1}, \eta_{0}\right)^{T}$.

We introduce the spaces

$L_{*}^{2}(0, l)=\left\{f \in L^{2}(0, l) ; f_{x}(0)=f_{x}(l)=0\right\}, \quad H_{*}^{m}(0, l)=H^{m}(0, l) \cap L_{*}^{2}(0, l)$.

The phase space $\mathcal{V}$ is given by $\mathcal{V}=\left[H_{0}^{1} \times L^{2}\right](0, l) \times L_{h}^{2}\left(0, \infty ; H_{0}^{1}(0, l)\right) \times\left[H_{*}^{1} \times L_{*}^{2}\right](0, l) \times\left(L_{h}^{2} \cap L_{g}^{2}\right)\left(0, \infty ; H_{0}^{1}(0, l)\right)$, where inner product for $U^{j}=\left(\varphi^{j}, \Phi^{j}, \nu^{j}, \psi^{j}, \Psi^{j}, \eta^{j}\right)^{T}, j=1,2$ given by

$$
\begin{aligned}
\left\langle U^{1}, U^{2}\right\rangle & =\rho_{1} \int_{0}^{l} \Phi^{1} \overline{\Phi^{2}} d x+\rho_{2} \int_{0}^{l} \Psi^{1} \overline{\Psi^{2}} d x \\
& +\kappa_{0} \int_{0}^{l}\left(\varphi_{x}^{1}+\psi^{1}\right) \overline{\left(\varphi_{x}^{2}+\psi^{2}\right)} d x+b_{0} \int_{0}^{l} \psi_{x}^{1} \overline{\psi_{x}^{2}} d x \\
& +\int_{0}^{l} \int_{0}^{\infty} h(s)\left(\nu_{x}^{1}+\eta^{1} \overline{\left(\nu_{x}^{2}+\eta^{2}\right)} d s d x\right. \\
& +\int_{0}^{l} \int_{0}^{\infty} g(s) \eta_{x}^{1} \overline{\eta_{x}^{2}} d s d x,
\end{aligned}
$$

with induced norm

$$
\begin{aligned}
\|U\|_{\mathcal{V}}^{2}= & \int_{0}^{l}\left[\rho_{1}|\Phi|^{2}+\rho_{2}|\Psi|^{2}+\kappa_{0}\left|\varphi_{x}+\psi\right|^{2}+b_{0}\left|\psi_{x}\right|^{2}\right. \\
& \left.+\int_{0}^{\infty} h(s)\left|\nu_{x}+\eta\right|^{2} d s+\int_{0}^{\infty} g(s)\left|\eta_{x}\right|^{2} d s\right] d x .
\end{aligned}
$$


Let $\mathcal{S}$ be the set bellow

$$
\left\{\kappa_{0}\left(\varphi_{x}+\psi\right)+\int_{0}^{\infty} h(s)\left(\nu_{x}+\eta\right)(s) d s \in H^{1}(0, l) ; b_{0} \psi+\int_{0}^{\infty} g(s) \eta(s) d s \in H_{*}^{2}(0, l)\right\} .
$$

From the above definitions, we introduce the domain of $\mathcal{A}$ by

$$
\begin{aligned}
D(\mathcal{A}) & =\left[H^{2}(0, l) \cap H_{0}^{1}(0, l)\right] \times H_{0}^{1}(0, l) \times H_{h}^{1}\left(0, \infty ; H_{0}^{1}(0, l)\right) \\
& \times H_{*}^{1}(0, l) \times H_{*}^{1}(0, l) \times\left(H_{h}^{1} \cap H_{g}^{1}\right)\left(0, \infty ; H_{0}^{1}(0, l)\right) \cap \mathcal{S} .
\end{aligned}
$$

We define the energy associated with the system (3.1)-(3.6) by

$$
\begin{aligned}
E(t):=\frac{1}{2} \int_{0}^{l} & {\left[\rho_{1}\left|\varphi_{t}\right|^{2}+\rho_{2}\left|\psi_{t}\right|^{2}+\kappa_{0}\left|\varphi_{x}+\psi\right|^{2}+b_{0}\left|\psi_{x}\right|^{2}+\right.} \\
& \left.+\int_{0}^{\infty} h(s)\left|\nu_{x}+\eta\right|^{2} d s+\int_{0}^{\infty} g(s)\left|\eta_{x}\right|^{2} d s\right] d x .
\end{aligned}
$$

In the sequel we have two important propositions.

Proposition 3.1. The operator $\mathcal{A}$ is dissipative.

Proof.

$$
\begin{aligned}
\langle\mathcal{A} U, U\rangle_{\mathcal{V}}= & \kappa_{0} \int_{0}^{l}\left(\varphi_{x}+\psi\right)_{x} \bar{\Phi} d x+\int_{0}^{l} \int_{0}^{\infty} h(s)\left(\nu_{x}+\eta\right)_{x}(x, s) d s \bar{\Phi} d x \\
& +b_{0} \int_{0}^{l} \psi_{x x} \bar{\Psi} d x-\kappa_{0} \int_{0}^{l}\left(\varphi_{x}+\psi\right) \bar{\Psi} d x \\
& +\int_{0}^{l} \int_{0}^{\infty} g(s) \eta_{x x}(x, s) d s \bar{\Psi} d x \\
& -\int_{0}^{l} \int_{0}^{\infty} h(s)\left(\nu_{x}+\eta\right)(x, s) d s \bar{\Psi} d x \\
& +\kappa_{0} \int_{0}^{l}\left(\Phi_{x}+\Psi\right) \overline{\left(\varphi_{x}+\psi\right)} d x+b_{0} \int_{0}^{l} \Psi_{x} \overline{\psi_{x}} d x \\
& +\int_{0}^{l} \int_{0}^{\infty} h(s)\left(\Phi_{x}-\nu_{s x}+\Psi-\eta_{s}\right) \overline{\left(\nu_{x}+\eta\right)} d s d x \\
& +\int_{0}^{l} \int_{0}^{\infty} g(s)\left(\Psi_{x}-\eta_{s x}\right) \overline{\eta_{x}} d s d x .
\end{aligned}
$$

Using boundary conditions (3.6) we obtain

$$
\begin{aligned}
\langle\mathcal{A} U, U\rangle_{\mathcal{V}}= & -\int_{0}^{\infty} h(s) \int_{0}^{l}\left(\nu_{x}+\eta\right)_{s} \overline{\left(\nu_{x}+\eta\right)} d x d s-\int_{0}^{\infty} g(s) \int_{0}^{l} \eta_{s x} \overline{\eta_{x}} d x d s \\
= & -\frac{1}{2} \int_{0}^{l}\left[\left.h(s)\left|\nu_{x}+\eta\right|^{2}\right|_{0} ^{\infty}-\int_{0}^{\infty} h^{\prime}(s)\left|\nu_{x}+\eta\right|^{2} d s\right] d x \\
& -\frac{1}{2} \int_{0}^{l}\left[\left.g(s)\left|\eta_{x}\right|^{2}\right|_{0} ^{\infty}-\int_{0}^{\infty} g^{\prime}(s)\left|\eta_{x}\right|^{2} d s\right] d x .
\end{aligned}
$$


Taking the real part we get

$\operatorname{Re}\langle\mathcal{A} U, U\rangle_{\mathcal{V}}=\frac{1}{2} \int_{0}^{\infty} h^{\prime}(s) \int_{0}^{l}\left|\nu_{x}+\eta\right|^{2} d x d s+\frac{1}{2} \int_{0}^{\infty} g^{\prime}(s) \int_{0}^{l}\left|\eta_{x}\right|^{2} d x d s$.

Then $\mathcal{A}$ is dissipative.

Proposition 3.2. $0 \in \rho(\mathcal{A})$, the resolvent set of $\mathcal{A}$.

Proof. The resolvent equation $\lambda U-\mathcal{A} U=F$, with $\lambda=0$ leads to

$$
\begin{aligned}
& -\Phi=f^{1} \in H_{0}^{1}(0, l), \\
& -\kappa_{0}\left(\varphi_{x}+\psi\right)_{x}-\int_{0}^{\infty} h(s)\left(\nu_{x}+\eta\right)_{x}(s) d s=\rho_{1} f^{2} \in L^{2}(0, l), \\
& -b_{0} \psi_{x x}+\kappa_{0}\left(\varphi_{x}+\psi\right)-\int_{0}^{\infty} g(s) \eta_{x x}(s) d s+\int_{0}^{\infty} h(s)\left(\nu_{x}+\eta\right)(s) d s=\rho_{2} f^{5} \in L_{*}^{2}(0, l),
\end{aligned}
$$

$$
-\Psi+\eta_{s}=f^{6} \in\left(L_{h}^{2} \cap L_{g}^{2}\right)\left(0, \infty ; H_{0}^{1}(0, l)\right) .
$$

From (3.7) and (3.10) follows, respectively, $\Phi \in H_{0}^{1}(0, l)$ and $\Psi \in H_{*}^{1}(0, l)$. From (3.9), we have $\nu_{s} \in L_{h}^{2}\left(0, \infty ; H_{0}^{1}(0, l)\right)$ and then $\nu \in H_{h}^{1}\left(0, \infty ; H_{0}^{1}(0, l)\right)$.

Similarly, from (3.11), we have $\eta_{s} \in\left(L_{h}^{2} \cap L_{g}^{2}\right)\left(0, \infty ; H_{0}^{1}(0, l)\right)$ and then $\eta \in\left(H_{h}^{1} \cap H_{g}^{1}\right)\left(0, \infty ; H_{0}^{1}(0, l)\right)$.

From (3.8) we have $\kappa_{0}\left(\varphi_{x}+\psi\right)+\int_{0}^{\infty} h(s)\left(\nu_{x}+\eta\right)(s) d s \in H^{1}(0, l)$ and then $b_{0} \psi+\int_{0}^{\infty} g(s) \eta(s) d s \in H_{*}^{2}(0, l)$.

Therefore, from definition of $D(\mathcal{A})$, follows that $U \in D(\mathcal{A})$ and then $0 \in \rho(\mathcal{A})$.

The existence of solution is given by the following theorem.

Theorem 3.1. For memory $h(t)$ and $g(t)$ as

$$
h(t)=\sum_{i=1}^{2} \mu_{i} e^{-\gamma_{i} t} \text { and } g(t)=\sum_{j=1}^{2} \nu_{j} e^{-\delta_{j} t},
$$

where $\mu_{i}, \nu_{j}, \gamma_{i}, \delta_{j}>0$ and $U_{0} \in D(\mathcal{A})$, then, there is a unique solution of system (3.1)-(3.4) with initial conditions (3.5) and boundary conditions (3.6) satisfying $U \in C\left(\mathbb{R}^{+} ; D(\mathcal{A})\right)$. Moreover, if $U_{0} \in D\left(\mathcal{A}^{n}\right)$, then $U \in$ $C^{n-k}\left(\mathbb{R}^{+} ; D\left(\mathcal{A}^{k}\right)\right), k=0,1, \ldots, n$.

Proof. By definition, $\mathcal{A}$ is a linear operator with dense domain $D(\mathcal{A})$ in the Hilbert space $\mathcal{V}$. As $\mathcal{A}$ is dissipative and $0 \in \rho(\mathcal{A})$, the resolvent set of $\mathcal{A}$, by Lummer-Phillips Theorem, (see [8], theorem 1.2.4), $\mathcal{A}$ is the infinitesimal generator of a $C_{0}$-semigroup of contractions $e^{\mathcal{A} t}$ on $\mathcal{V}$. Then, defining $U(t)=$ $e^{\mathcal{A} t} U_{0}$ as a direct consequence of semigroup theory, (see [9]), we have that 
$U(t)$ is the unique solution of (3.1)-(3.4) in the class $U \in C\left(\mathbb{R}^{+} ; D(\mathcal{A})\right)$. Moreover, if $U_{0} \in D\left(\mathcal{A}^{n}\right)$, then $U \in C^{n-k}\left(\mathbb{R}^{+} ; D\left(\mathcal{A}^{k}\right)\right), k=0,1, \ldots, n$.

\section{Property of Growth Determined by Spectrum}

In this section we prove the property of growth determined by the spectrum of the operator analyzing the memory in three different situations.

4.1. Linear stability when $h(t)=\mu e^{-\gamma t}, \mu, \gamma>0$. Consider the Timoshenko system with memory on the cutting

$$
\begin{aligned}
\rho_{1} \varphi_{t t}-S_{x} & =0, \text { in }(0, l) \times(0, t), \\
\rho_{2} \psi_{t t}-M_{x}+S & =0, \text { in }(0, l) \times(0, t),
\end{aligned}
$$

where

$$
S=\kappa\left(\varphi_{x}+\psi\right)-\int_{-\infty}^{t} h(t-s)\left(\varphi_{x}+\psi\right)(x, s) d s
$$

and

$$
M=b \psi_{x} .
$$

Then, we have in $(0, l) \times(0, t)$

$$
\rho_{1} \varphi_{t t}-\kappa\left(\varphi_{x}+\psi\right)_{x}+\int_{-\infty}^{t} h(t-s)\left(\varphi_{x}+\psi\right)_{x}(s) d s=0,
$$

$$
\rho_{2} \psi_{t t}-b \psi_{x x}+\kappa\left(\varphi_{x}+\psi\right)-\int_{-\infty}^{t} h(t-s)\left(\varphi_{x}+\psi\right)(s) d s=0,
$$

with the initial conditions

$$
\varphi(x, 0)=\varphi_{0}(x), \varphi_{t}(x, 0)=\varphi_{1}(x), \psi(x, 0)=\psi_{0}(x), \psi_{t}(x, 0)=\psi_{1}(x),
$$

and boundary conditions

$$
\varphi(0, t)=\varphi(l, t)=\psi_{x}(0, t)=\psi_{x}(l, t)=0 .
$$

Making $U=\left(\varphi, \varphi_{t}, \psi, \psi_{t},\right)^{T}$, we get

$$
U_{t}=\left(\begin{array}{c}
\varphi_{t} \\
\varphi_{t t} \\
\psi_{t} \\
\psi_{t t}
\end{array}\right)=\underbrace{\left(\begin{array}{c}
\varphi_{t} \\
\frac{\kappa}{\rho_{1}}\left(\varphi_{x}+\psi\right)_{x} \\
\psi_{t} \\
\frac{b}{\rho_{2}} \psi_{x x}-\frac{\kappa}{\rho_{2}}\left(\varphi_{x}+\psi\right)
\end{array}\right)}_{:=\mathcal{A}_{0} U}-\underbrace{h *\left(\begin{array}{c}
0 \\
\frac{1}{\rho_{1}}\left(\varphi_{x}+\psi\right)_{x} \\
0 \\
\frac{1}{\rho_{2}}\left(\varphi_{x}+\psi\right)
\end{array}\right)}_{:=h * \mathcal{A}_{1} U},
$$

where $h * f$ denotes the convolution of the type $\int_{-\infty}^{t} h(t-s) f(s) d s$.

So, we have

$$
U_{t}=\mathcal{A}_{0} U-h * \mathcal{A}_{1} U \text {. }
$$

Deriving we have

$$
U_{t t}=\mathcal{A}_{0} U_{t}-h^{\prime} * \mathcal{A}_{1} U=\mathcal{A}_{0} U_{t}+\gamma h * \mathcal{A}_{1} U .
$$


Then, we obtain

$$
U_{t t}=\mathcal{A}_{0} U_{t}+B
$$

where $B=-\gamma U_{t}+\gamma \mathcal{A}_{0} U$.

Theorem 4.1. The system (4.1)-(4.3) verifies the property of growth determined by spectrum.

Proof. In this case we consider the infinitesimal generator for the Timoshenko system,

$$
\begin{aligned}
\rho_{1} \varphi_{t t}-\kappa\left(\varphi_{x}+\psi\right)_{x} & =0 \\
\rho_{2} \psi_{t t}-b \psi_{x x}+\kappa\left(\varphi_{x}+\psi\right) & =0
\end{aligned}
$$

then,

$$
\mathcal{A}_{0}=\left(\begin{array}{cccc}
0 & I & 0 & 0 \\
\frac{\kappa}{\rho_{1}} \partial_{x}^{2} & 0 & \frac{\kappa}{\rho_{1}} \partial_{x} & 0 \\
0 & 0 & 0 & I \\
-\frac{\kappa}{\rho_{2}} \partial_{x} & 0 & \frac{b}{\rho_{2}} \partial_{x}^{2}-\frac{\kappa}{\rho_{2}} I & 0
\end{array}\right), \quad U=\left(\begin{array}{c}
\varphi \\
\varphi_{t} \\
\psi \\
\psi_{t}
\end{array}\right)
$$

Note that $\mathcal{A}_{0}$ is a normal operator. So, the problem (4.4)-(4.5) can be rewrite as

$$
U_{t}-\mathcal{A}_{0} U=0 \text {. }
$$

Therefore, the model associate with the Cauchy problem (4.1)-(4.3) is defined by

$$
\mathcal{U}_{t}-\mathcal{A U}=\mathcal{F}
$$

where

$$
\mathcal{A}=\left(\begin{array}{ccc}
0 & I & 0 \\
0 & 0 & I \\
0 & 0 & \mathcal{A}_{0}
\end{array}\right), \quad \mathcal{F}=\left(\begin{array}{c}
0 \\
0 \\
B_{t}
\end{array}\right), \quad \mathcal{U}=\left(\begin{array}{c}
U \\
U_{t} \\
U_{t t}
\end{array}\right) .
$$

As $\mathcal{A}_{0}$ is normal, from Theorem 2.1 follows that $\mathcal{A}$ is a normal operator that verifies the conditions (1) and (2).

The continuity of $B_{t}$ is made through derived inequalities. Using the Theorem 1.1 the result follows.

4.2. Linear stability when $g(t)=\nu e^{-\delta t}, \nu, \delta>0$. Consider the system memory on the flexor moment

$$
\begin{aligned}
\rho_{1} \varphi_{t t}-S_{x} & =0, \text { in }(0, l) \times(0, t), \\
\rho_{2} \psi_{t t}-M_{x}+S & =0, \text { in }(0, l) \times(0, t),
\end{aligned}
$$

where

$$
S=\kappa\left(\varphi_{x}+\psi\right)
$$

and

$$
M=b \psi_{x}-\int_{-\infty}^{t} g(t-s) \psi_{x}(x, s) d s
$$


In $(0, l) \times(0, t)$, the moment like this form

$$
\rho_{1} \varphi_{t t}-\kappa\left(\varphi_{x}+\psi\right)_{x}=0
$$

$\rho_{2} \psi_{t t}-b \psi_{x x}+\int_{-\infty}^{t} g(t-s) \psi_{x x}(s) d s+\kappa\left(\varphi_{x}+\psi\right)=0$, in $(0, l) \times(0, t)$.

The initial conditions are given by

$$
\varphi(x, 0)=\varphi_{0}(x), \varphi_{t}(x, 0)=\varphi_{1}(x), \psi(x, 0)=\psi_{0}(x), \psi_{t}(x, 0)=\psi_{1}(x),
$$

and the boundary conditions type Dirichlet-Neumann are

$$
\varphi(0, t)=\varphi(l, t)=\psi_{x}(0, t)=\psi_{x}(l, t)=0 .
$$

Making $U=\left(\varphi, \varphi_{t}, \psi, \psi_{t},\right)^{T}$, we have

$$
U_{t}=\left(\begin{array}{c}
\varphi_{t} \\
\varphi_{t t} \\
\psi_{t} \\
\psi_{t t}
\end{array}\right)=\underbrace{\left(\begin{array}{c}
\varphi_{t} \\
\frac{\kappa}{\rho_{1}}\left(\varphi_{x}+\psi\right)_{x} \\
\psi_{t} \\
\frac{b}{\rho_{2}} \psi_{x x}-\frac{\kappa}{\rho_{2}}\left(\varphi_{x}+\psi\right)
\end{array}\right)}_{:=\mathcal{A}_{0} U}-g * \underbrace{\left(\begin{array}{c}
0 \\
0 \\
0 \\
\frac{1}{\rho_{2}} \psi_{x x}
\end{array}\right)}_{:=g * \mathcal{A}_{2} U},
$$

where $g * f$ denotes the convolution $\int_{-\infty}^{t} g(t-s) f(s) d s$.

So we have

$$
U_{t}=\mathcal{A}_{0} U-g * \mathcal{A}_{2} U
$$

Deriving,

$$
U_{t t}=\mathcal{A}_{0} U_{t}-g^{\prime} * \mathcal{A}_{2} U=\mathcal{A}_{0} U_{t}+\delta g * \mathcal{A}_{2} U
$$

So, we obtain

$$
U_{t t}=\mathcal{A}_{0} U_{t}-\delta U_{t}+\delta \mathcal{A}_{0} U
$$

Consider $C=-\delta U_{t}+\delta \mathcal{A}_{0} U$.

Theorem 4.2. The system (4.6)-(4.7) verifies the property of linear stability.

Proof. In this case, we consider the infinitesimal generate as

$$
\begin{array}{r}
\rho_{1} \varphi_{t t}-\kappa\left(\varphi_{x}+\psi\right)_{x}=0, \\
\rho_{2} \psi_{t t}-b \psi_{x x}+\kappa\left(\varphi_{x}+\psi\right)=0,
\end{array}
$$

then

$$
\mathcal{A}_{0}=\left(\begin{array}{cccc}
0 & I & 0 & 0 \\
\frac{\kappa}{\rho_{1}} \partial_{x}^{2} & 0 & \frac{\kappa}{\rho_{1}} \partial_{x} & 0 \\
0 & 0 & 0 & I \\
-\frac{\kappa}{\rho_{2}} \partial_{x} & 0 & \frac{b}{\rho_{2}} \partial_{x}^{2}-\frac{\kappa}{\rho_{2}} I & 0
\end{array}\right), \quad U=\left(\begin{array}{c}
\varphi \\
\varphi_{t} \\
\psi \\
\psi_{t}
\end{array}\right) .
$$

Note that $\mathcal{A}_{0}$ is a normal operator. So, the problem (4.8)-(4.9) can be rewrite as

$$
U_{t}-\mathcal{A}_{0} U=0
$$


Then, the associated Cauchy problem (4.6)-(4.7) is defined by

$$
\mathcal{U}_{t}-\mathcal{A U}=\mathcal{G},
$$

where

$$
\mathcal{A}=\left(\begin{array}{ccc}
0 & I & 0 \\
0 & 0 & I \\
0 & 0 & \mathcal{A}_{0}
\end{array}\right), \quad \mathcal{G}=\left(\begin{array}{c}
0 \\
0 \\
C_{t}
\end{array}\right), \quad \mathcal{U}=\left(\begin{array}{c}
U \\
U_{t} \\
U_{t t}
\end{array}\right) .
$$

As $\mathcal{A}_{0}$ is normal, from Theorem 2.1 follows that $\mathcal{A}$ is a normal operator that verifies the conditions (1) and (2).

The continuity of $C_{t}$ is made through derivative inequalities. By Theorem 1.1 , the result follows.

4.3. Linear Stability when $h(t)=\mu e^{-\gamma t}$ and $g(t)=\nu e^{-\delta t}, \mu, \nu, \gamma, \delta>0$. Now consider the case with two memory

$$
\begin{aligned}
\rho_{1} \varphi_{t t}-S_{x}=0, & \text { in }(0, l) \times(0, t), \\
\rho_{2} \psi_{t t}-M_{x}+S=0, & \text { in }(0, l) \times(0, t),
\end{aligned}
$$

where

$$
S=\kappa\left(\varphi_{x}+\psi\right)-\int_{-\infty}^{t} h(t-s)\left(\varphi_{x}+\psi\right)(x, s) d s
$$

and

$$
M=b \psi_{x}-\int_{-\infty}^{t} g(t-s) \psi_{x}(x, s) d s
$$

In $(0, l) \times(0, t)$ the model now is

$$
\begin{array}{r}
\rho_{2} \psi_{t t}-b \psi_{x x}+\int_{-\infty}^{t} g(t-s) \psi_{x x}(s) d s \\
+\kappa\left(\varphi_{x}+\psi\right)-\int_{-\infty}^{t} h(t-s)\left(\varphi_{x}+\psi\right)(s) d s=0 .
\end{array}
$$

The initial conditions are given by

$$
\varphi(x, 0)=\varphi_{0}(x), \varphi_{t}(x, 0)=\varphi_{1}(x), \psi(x, 0)=\psi_{0}(x), \psi_{t}(x, 0)=\psi_{1}(x),
$$

and boundary conditions type Dirichlet-Neumann are

$$
\varphi(0, t)=\varphi(l, t)=\psi_{x}(0, t)=\psi_{x}(l, t)=0 .
$$


Making $U=\left(\varphi, \varphi_{t}, \psi, \psi_{t},\right)^{T}$, we obtain

$$
U_{t}=\underbrace{\left(\begin{array}{c}
\varphi_{t} \\
\frac{\kappa}{\rho_{1}}\left(\varphi_{x}+\psi\right)_{x} \\
\psi_{t} \\
\frac{b}{\rho_{2}} \psi_{x x}-\frac{\kappa}{\rho_{2}}\left(\varphi_{x}+\psi\right)
\end{array}\right)}_{:=\mathcal{A}_{0} U}-h * \underbrace{\left(\begin{array}{c}
0 \\
\frac{1}{\rho_{1}}\left(\varphi_{x}+\psi\right)_{x} \\
0 \\
\frac{1}{\rho_{2}}\left(\varphi_{x}+\psi\right)
\end{array}\right)}_{:=h * \mathcal{A}_{1} U}-g * \underbrace{\left(\begin{array}{c}
0 \\
0 \\
0 \\
0 \\
\frac{1}{\rho_{2}} \psi_{x x}
\end{array}\right)}_{:=g * \mathcal{A}_{2} U}
$$

where $h * f$ and $g * f$ denote the convolutions

$$
\int_{-\infty}^{t} h(t-s) f(s) d s \text { and } \int_{-\infty}^{t} g(t-s) f(s) d s .
$$

So, we have

$$
U_{t}=\mathcal{A}_{0} U-h * \mathcal{A}_{1} U-g * \mathcal{A}_{2} U .
$$

After to derive we obtain

$$
U_{t t}=\mathcal{A}_{0} U_{t}-h^{\prime} * \mathcal{A}_{1} U-g^{\prime} * \mathcal{A}_{2} U=\mathcal{A}_{0} U_{t}+\gamma h * \mathcal{A}_{1} U+\delta g * \mathcal{A}_{2} U .
$$

Now defining the operator $\mathcal{L}: H \rightarrow H$, where $H$ are Hilbert spaces, given by

$$
\mathcal{L}(f)=f^{\prime \prime}+(\gamma+\delta) f^{\prime}+\gamma \delta f
$$

Clearly we have

$$
\mathcal{L}(h(t))=0 \text { and } \mathcal{L}(g(t))=0 .
$$

Applying $\mathcal{L}$ in the equation (4.14), we have

$$
\mathcal{L}\left(U_{t}\right)=\mathcal{A}_{0} \mathcal{L}(U)-\mathcal{L}(h) * \mathcal{A}_{1} U-\mathcal{L}(g) * \mathcal{A}_{2} U .
$$

So, we have

$$
U_{t t t}+(\gamma+\delta) U_{t t}+\gamma \delta U_{t}=\mathcal{A}_{0} U_{t t}+(\gamma+\delta) \mathcal{A}_{0} U_{t}+\gamma \delta \mathcal{A}_{0} U
$$

then

$$
U_{t t t}=\mathcal{A}_{0} U_{t t}-(\gamma+\delta) U_{t t}-\gamma \delta U_{t}+(\gamma+\delta) \mathcal{A}_{0} U_{t}+\gamma \delta \mathcal{A}_{0} U
$$

Now consider $D=-(\gamma+\delta) U_{t t}-\gamma \delta U_{t}+(\gamma+\delta) \mathcal{A}_{0} U_{t}+\gamma \delta \mathcal{A}_{0} U$.

Theorem 4.3. The system (4.10)-(4.13) verifies the linear stability.

Proof. For this case we consider the following infinitesimal generator

$$
\begin{aligned}
\rho_{1} \varphi_{t t}-\kappa\left(\varphi_{x}+\psi\right)_{x} & =0 \\
\rho_{2} \psi_{t t}-b \psi_{x x}+\kappa\left(\varphi_{x}+\psi\right) & =0
\end{aligned}
$$

then

$$
\mathcal{A}_{0}=\left(\begin{array}{cccc}
0 & I & 0 & 0 \\
\frac{\kappa}{\rho_{1}} \partial_{x}^{2} & 0 & \frac{\kappa}{\rho_{1}} \partial_{x} & 0 \\
0 & 0 & 0 & I \\
-\frac{\kappa}{\rho_{2}} \partial_{x} & 0 & \frac{b}{\rho_{2}} \partial_{x}^{2}-\frac{\kappa}{\rho_{2}} I & 0
\end{array}\right), \quad U=\left(\begin{array}{c}
\varphi \\
\varphi_{t} \\
\psi \\
\psi_{t}
\end{array}\right)
$$


Note that $\mathcal{A}_{0}$ is a normal operator. So, the problem (4.15)-(4.16) can be rewrite as

$$
U_{t}-\mathcal{A}_{0} U=0 .
$$

Hence the model associated to Cauchy problem (4.10)-(4.13) is defined by

$$
\mathcal{U}_{t}-\mathcal{A U}=\mathcal{J},
$$

where

$$
\mathcal{A}=\left(\begin{array}{ccc}
0 & I & 0 \\
0 & 0 & I \\
0 & 0 & \mathcal{A}_{0}
\end{array}\right), \quad \mathcal{J}=\left(\begin{array}{c}
0 \\
0 \\
D
\end{array}\right), \quad \mathcal{U}=\left(\begin{array}{c}
U \\
U_{t} \\
U_{t t}
\end{array}\right) .
$$

As $\mathcal{A}_{0}$ is normal, from Theorem 2.1 follows that $\mathcal{A}$ is a normal operator that verifies the conditions (1) and (2).

The continuity of $D$ is made through derivative inequalities. By Theorem 1.1 we obtain the result.

\section{Calculation of $\omega_{0}(\mathcal{A})$}

In this section we will calculate the polynomial whose roots give us the estimate of $\omega_{0}(\mathcal{A})$. Denote, as in Section 4.1, the operator $\mathcal{A}$ given by

$$
\mathcal{A} U=\left[\begin{array}{c}
\Phi \\
\frac{\kappa_{0}}{\rho_{1}}\left(\varphi_{x}+\psi\right)_{x}+\frac{1}{\rho_{1}} \int_{0}^{\infty} h(s)\left(\nu_{x}+\eta\right)_{x}(s) d s \\
\Phi-\nu_{s} \\
\Psi \\
\frac{b_{0}}{\rho_{2}} \psi_{x x}-\frac{\kappa_{0}}{\rho_{2}}\left(\varphi_{x}+\psi\right)+\frac{1}{\rho_{2}} \int_{0}^{\infty} g(s) \eta_{x x}(s) d s-\frac{1}{\rho_{2}} \int_{0}^{\infty} h(s)\left(\nu_{x}+\eta\right)(s) d s \\
\Psi-\eta_{s}
\end{array}\right] .
$$

We denote by $V$ the space

$V=\left[H_{0}^{1} \times L^{2}\right](0, l) \times L_{h}^{2}\left(0, \infty ; H_{0}^{1}(0, l)\right) \times\left[H_{*}^{1} \times L_{*}^{2}\right](0, l) \times\left(L_{h}^{2} \cap L_{g}^{2}\right)\left(0, \infty ; H_{0}^{1}(0, l)\right)$, where

$$
L_{*}^{2}(0, l)=\left\{f \in L^{2}(0, l) ; f_{x}(0)=f_{x}(l)=0\right\}, H_{*}^{m}(0, l)=H^{m}(0, l) \cap L_{*}^{2}(0, l)
$$

and

$$
L_{\mu}^{2}\left(0, \infty ; H_{0}^{1}(0, l)\right)=\left\{f \in H_{0}^{1}(0, l) ; \int_{0}^{+\infty} \mu(s) \int_{0}^{l}\left|f_{x}(x, s)\right|^{2} d x d s<\infty\right\},
$$

with induced norm

$$
\begin{aligned}
\|U\|_{V}^{2} & =\int_{0}^{l}\left[\rho_{1}|\Phi|^{2}+\rho_{2}|\Psi|^{2}+\kappa_{0}\left|\varphi_{x}+\psi\right|^{2}+b_{0}\left|\psi_{x}\right|^{2}\right. \\
& \left.+\int_{0}^{\infty} h(s)\left|\nu_{x}+\eta\right|^{2} d s+\int_{0}^{\infty} g(s)\left|\eta_{x}\right|^{2} d s\right] d x .
\end{aligned}
$$


The domain $D(\mathcal{A})$ of $\mathcal{A}$ is given by

$$
\begin{aligned}
D(\mathcal{A}) & =\left[H^{2}(0, l) \cap H_{0}^{1}(0, l)\right] \times H_{0}^{1}(0, l) \times H_{h}^{1}\left(0, \infty ; H_{0}^{1}(0, l)\right) \\
& \times H_{*}^{1}(0, l) \times H_{*}^{1}(0, l) \times\left(H_{h}^{1} \cap H_{g}^{1}\right)\left(0, \infty ; H_{0}^{1}(0, l)\right) \cap \mathcal{S},
\end{aligned}
$$

where $\mathcal{S}$ is the set bellow

$\left\{\kappa_{0}\left(\varphi_{x}+\psi\right)+\int_{0}^{\infty} h(s)\left(\nu_{x}+\eta\right)(s) d s \in H^{1}(0, l) ; b_{0} \psi+\int_{0}^{\infty} g(s) \eta(s) d s \in H_{*}^{2}(0, l)\right\}$.

The resolvent equation

$$
\lambda U-\mathcal{A} U=F,
$$

in terms of the scalar components, is given by

$$
\begin{gathered}
\lambda \varphi-\Phi=f^{1} \in H_{0}^{1}(0, l), \\
\lambda \rho_{1} \Phi-\kappa_{0}\left(\varphi_{x}+\psi\right)_{x}-\int_{0}^{\infty} h(s)\left(\nu_{x}+\eta\right)_{x} d s=f^{2} \in L^{2}(0, l), \\
\lambda \nu-\Phi+\nu_{s}=f^{3} \in L_{h}^{2}\left(0, \infty ; H_{0}^{1}(0, l)\right), \\
\lambda \psi-\Psi=f^{4} \in H_{*}^{1}(0, l), \\
\lambda \rho_{2} \Psi-b_{0} \psi_{x x}+\kappa_{0}\left(\varphi_{x}+\psi\right) \\
-\int_{0}^{\infty}(s) \eta_{x x}(s) d s+\int_{0}^{\infty} h(s)\left(\nu_{x}+\eta\right)(s) d s=f^{5} \in L_{*}^{2}(0, l), \\
\lambda \eta-\Psi+\eta_{s}=f^{6} \in\left(L_{h}^{2} \cap L_{g}^{2}\right)\left(0, \infty ; H_{0}^{1}(0, l)\right) .
\end{gathered}
$$

We must find the elements of the spectrum of operator. It's simple verifies that $\lambda \in \sigma(\mathcal{A})$ if and only if

$$
\lambda U-\mathcal{A} U=0 .
$$

From (5.1)-(5.6) for $f^{i}=0$, for all $i=\{1, \ldots, 6\}$, we find

$$
\Phi=\lambda \varphi \quad \text { and } \quad \Psi=\lambda \psi
$$

then

$$
\begin{gathered}
\lambda^{2} \rho_{1} \varphi-\kappa_{0}\left(\varphi_{x}+\psi\right)_{x}-\int_{0}^{\infty} h(s)\left(\nu_{x}+\eta\right)_{x} d s=0, \\
\lambda \nu-\lambda \varphi+\nu_{s}=0, \\
\lambda^{2} \rho_{2} \psi-b_{0} \psi_{x x}+\kappa_{0}\left(\varphi_{x}+\psi\right) \\
-\int_{0}^{\infty} g(s) \eta_{x x}(s) d s+\int_{0}^{\infty} h(s)\left(\nu_{x}+\eta\right)(s) d s=0, \\
\lambda \eta-\lambda \psi+\eta_{s}=0 .
\end{gathered}
$$

Solving the equations (5.8) and (5.10), we have

$$
\nu_{s}+\lambda \nu=\lambda \varphi \text { and } \eta_{s}+\lambda \eta=\lambda \psi,
$$


that is

$$
\left[e^{\lambda s} \nu\right]_{s}=\lambda e^{\lambda s} \varphi \text { and }\left[e^{\lambda s} \eta\right]_{s}=\lambda e^{\lambda s} \psi .
$$

From where follows that

$$
e^{\lambda s} \nu=\int_{0}^{s} \lambda e^{\lambda \tau} \varphi(x, t) d \tau \text { and } e^{\lambda s} \eta=\int_{0}^{s} \lambda e^{\lambda \tau} \psi(x, t) d \tau .
$$

Calculating the integrals, we have

$$
e^{\lambda s} \nu=\left.e^{\lambda \tau}\right|_{0} ^{s} \varphi(x, t)=\left(e^{\lambda s}-1\right) \varphi \quad \text { and } \quad e^{\lambda s} \eta=\left.e^{\lambda \tau}\right|_{0} ^{s} \psi(x, t)=\left(e^{\lambda s}-1\right) \psi
$$

So,

$$
\nu=\left(1-e^{-\lambda s}\right) \varphi \quad \text { e } \quad \eta=\left(1-e^{-\lambda s}\right) \psi .
$$

Replacing $\nu$ and $\eta$ in (5.7) and (5.9), we obtain

$$
\lambda^{2} \rho_{1} \varphi-\kappa_{0}\left(\varphi_{x}+\psi\right)_{x}-\left(\varphi_{x}+\psi\right)_{x} \int_{0}^{\infty} h(s)\left(1-e^{-\lambda s}\right) d s=0
$$

and

$$
\begin{array}{r}
\lambda^{2} \rho_{2} \psi-b_{0} \psi_{x x}+\kappa_{0}\left(\varphi_{x}+\psi\right)-\psi_{x x} \int_{0}^{\infty} g(s)\left(1-e^{-\lambda s}\right) d s+ \\
+\left(\varphi_{x}+\psi\right) \int_{0}^{\infty} h(s)\left(1-e^{-\lambda s}\right) d s=0 .
\end{array}
$$

Suppose $h(t)=\mu e^{-\gamma t}$ and $g(t)=0$.

In this case, the equations (5.11)-(5.12) remain

$$
\lambda^{2} \rho_{1} \varphi-\kappa_{0}\left(\varphi_{x}+\psi\right)_{x}-\left(\varphi_{x}+\psi\right)_{x} \int_{0}^{\infty} h(s)\left(1-e^{-\lambda s}\right) d s=0
$$

and

$$
\lambda^{2} \rho_{2} \psi-b_{0} \psi_{x x}+\kappa_{0}\left(\varphi_{x}+\psi\right)+\left(\varphi_{x}+\psi\right) \int_{0}^{\infty} h(s)\left(1-e^{-\lambda s}\right) d s=0 .
$$

Differentiating (5.14) in relation $x$, we have

$$
\begin{aligned}
& \lambda^{2} \rho_{2} \psi_{x}-b_{0} \psi_{x x x}+\kappa_{0}\left(\varphi_{x}+\psi\right)_{x}+\left(\varphi_{x}\right. \\
& +\psi)_{x} \int_{0}^{\infty} h(s)\left(1-e^{-\lambda s}\right) d s=0 .
\end{aligned}
$$

From (5.13), follows

$$
\kappa_{0}\left(\varphi_{x}+\psi\right)_{x}+\left(\varphi_{x}+\psi\right)_{x} \int_{0}^{\infty} h(s)\left(1-e^{-\lambda s}\right) d s=\lambda^{2} \rho_{1} \varphi .
$$

Replacing (5.16) in (5.15), we obtain

$$
\lambda^{2} \rho_{2} \psi_{x}-b_{0} \psi_{x x x}+\lambda^{2} \rho_{1} \varphi=0 .
$$


By outer side, using the boundary conditions (4.12), we obtain $\varphi$ and $\psi$ of type

$$
\varphi(x, t)=e^{\lambda t} \sin (\widetilde{\gamma} x) \text { and } \psi(x, t)=e^{\lambda t} \cos (\widetilde{\gamma} x),
$$

where $\widetilde{\gamma}$ is such that $\widetilde{\gamma} l=n \pi, n \in \mathbb{N}$.

Estimating the derivatives of $\psi$, we get

$$
\psi_{x}=-\widetilde{\gamma} \varphi, \quad \psi_{x x}=-\widetilde{\gamma}^{2} \psi \text { and } \psi_{x x x}=\widetilde{\gamma}^{3} \varphi .
$$

Replacing in (5.17) and, simplifying by $\varphi \neq 0$, follows that

$$
\lambda^{2}\left(\rho_{1}-\rho_{2} \widetilde{\gamma}\right)-b_{0} \widetilde{\gamma}^{3}=0 .
$$

Let $P_{1}(\lambda)$ be the polynomial of degree 2 given by

$$
P_{1}(\lambda)=\lambda^{2}\left(\rho_{1}-\frac{\rho_{2} n \pi}{l}\right)-b_{0} \frac{n^{3} \pi^{3}}{l^{3}}, n \in \mathbb{N} .
$$

We consider

$$
\sigma(\mathcal{A})=\left\{\lambda \in \mathbb{C} / P_{1}(\lambda)=0\right\} .
$$

Solving $P_{1}(\lambda)=0$, we obtain

$$
\lambda^{2}=\frac{b_{0} n^{3} \pi^{3}}{l^{2}\left(l \rho_{1}-\rho_{2} n \pi\right)}, n \in \mathbb{N} .
$$

So,

$$
\lambda= \pm i \sqrt{\frac{b_{0} n^{3} \pi^{3}}{l^{2}\left(l \rho_{1}-\rho_{2} n \pi\right)}}, n \in \mathbb{N} .
$$

Then

$$
\omega_{0}(\mathcal{A})=\sup \{\mathcal{R} e \lambda ; \lambda \in \sigma(\mathcal{A})\}
$$

Suppose $g(t)=\nu e^{-\delta t}$ and $h(t)=0$.

Now, the equations (5.11)-(5.12) remain

$$
\lambda^{2} \rho_{1} \varphi-\kappa_{0}\left(\varphi_{x}+\psi\right)_{x}=0
$$

and

$$
\lambda^{2} \rho_{2} \psi-b_{0} \psi_{x x}+\kappa_{0}\left(\varphi_{x}+\psi\right)-\psi_{x x} \int_{0}^{\infty} g(s)\left(1-e^{-\lambda s}\right) d s=0 .
$$

Differentiating the last equation in relation $x$, we get

$$
\begin{aligned}
& \lambda^{2} \rho_{2} \psi_{x}-b_{0} \psi_{x x x}+\kappa_{0}\left(\varphi_{x}+\psi\right)_{x} \\
& -\psi_{x x x} \int_{0}^{\infty} g(s)\left(1-e^{-\lambda s}\right) d s=0 .
\end{aligned}
$$

From (5.18), follows that

$$
\kappa_{0}\left(\varphi_{x}+\psi\right)_{x}=\lambda^{2} \rho_{1} \varphi .
$$


Replacing (5.20) in (5.19), we get

$$
\lambda^{2} \rho_{2} \psi_{x}-b_{0} \psi_{x x x}+\lambda^{2} \rho_{1} \varphi-\psi_{x x x} \int_{0}^{\infty} g(s)\left(1-e^{-\lambda s}\right) d s=0
$$

Moreover, using boundary conditions (4.12) we get $\varphi$ and $\psi$ type

$$
\varphi(x, t)=e^{\lambda t} \sin (\widetilde{\gamma} x) \text { and } \psi(x, t)=e^{\lambda t} \cos (\widetilde{\gamma} x),
$$

where $\widetilde{\gamma}$ is such that $\widetilde{\gamma} l=n \pi, n \in \mathbb{N}$.

From estimate of derived $\psi$, we have

$$
\psi_{x}=-\widetilde{\gamma} \varphi, \quad \psi_{x x}=-\widetilde{\gamma}^{2} \psi, \quad \psi_{x x x}=\widetilde{\gamma}^{3} \varphi .
$$

Using the dissipative mechanism $g(t)=\nu e^{-\delta t}$, where $\nu, \delta>0$, replacing in (5.21) and simplifying by $\varphi \neq 0$, follows that

$$
\lambda^{2}\left(\rho_{1}-\rho_{2} \widetilde{\gamma}\right)-b_{0} \widetilde{\gamma}^{3}-\widetilde{\gamma}^{3} \nu \int_{0}^{\infty} e^{-\delta s}\left(1-e^{-\lambda s}\right) d s=0 .
$$

Calculating the integral, we obtain

$$
\lambda^{2}\left(\rho_{1}-\rho_{2} \widetilde{\gamma}\right)-b_{0} \widetilde{\gamma}^{3}-\widetilde{\gamma}^{3} \nu\left[\frac{e^{-\delta s}}{-\delta}+\frac{e^{-(\delta+\lambda) s}}{-(\delta+\lambda)}\right]_{0}^{\infty}=0 .
$$

Consider the polynomial $P_{2}(\lambda)$ of degree 2 given by

$$
P_{2}(\lambda)=\lambda^{2}\left(\rho_{1}-\rho_{2} \widetilde{\gamma}\right)-b_{0} \widetilde{\gamma}^{3}-\widetilde{\gamma}^{3} \nu\left(\frac{1}{\delta}+\frac{1}{\delta+\lambda}\right) .
$$

Proceeding as before, we get

$$
\sigma(\mathcal{A})=\left\{\lambda \in \mathbb{C} / P_{2}(\lambda)=0\right\},
$$

and solving $P_{2}(\lambda)=0$, we obtain

$$
\omega_{0}(\mathcal{A})=\sup \{\mathcal{R} e \lambda ; \lambda \in \sigma(\mathcal{A})\} .
$$

\section{COnClusion}

The property of growth determined by spectrum $w_{\sigma}(A)=w_{0}(A)$ gives an important information, that is, the best constant for the exponential stability is the upper bound of the spectrum of operator $A$. In this sense, we prove that the Timoshenko system with memory acting on both, bending moment and shear force, is exponentially stable with optimal rate.

\section{REFERENCES}

[1] R. A. Adams, Sobolev Spaces, Academic Press, New York, 1975.

[2] C. M. Dafermos, On abstract Volterra equation with applications to linear viscoelasticity, Differential and Integral Equations, 7 (1970), 554-569.

[3] C. M. Dafermos, Asymptotic Stability in Viscoelasticity, Arch. Rat. Mech. Anal., 37 (1970), 297-308.

[4] M. Fabrizio, A. Morro, Mathematical problems in linear viscoelasticity, SIAM - Studies in Applied Mathematics, 12, Philadelphia, 1992. 
[5] B. Feng, Ma Tu Fu, R. N. Monteiro, C. A. Raposo, Dynamics of Laminated Timoshenko Beams Journal of Dynamics and Differential Equations, 30 (2018), 1489-1507.

[6] S. A. Messaoudi, B. Said-Houari, Uniform decay in a Timoshenko-type system with past history, J. Math. Anal. Appl., 360 (2009), 459-475.

[7] K. Liu, Z. Liu, On the type of $C_{0}$-semigroups associated with the abstract linear viscoelastic system, Z. Angew. Math. Phys., 47 (1996), 1-15.

[8] Z. Liu, S. Zheng, Semigroups associated with dissipative systems, Chapman \& Hall/CRC, 1999.

[9] A. Pazy, Semigroup of linear operators and applications to partial differential equations, Springer-Verlag, New York, 1983.

[10] J. Prüss, On the Spectrum of $C_{0}$-Semigroups, Transaction of the American Mathematical Society, 284 (1984), 847-857.

[11] C. A. Raposo, J. E. M. Rivera, R. R. Alves, Property of growth determined by spectrum of operator associated with the Timoshenko system with weakly dissipation Differ. Equ. Appl., 7 (2015), 385-400.

[12] C. A. Raposo, O. Vera Villagran, J. E. Munoz Rivera, M. Alves, Hybrid laminated Timoshenko beam, J. Math. Phys., 58 (2017), 101512.

[13] M. Renardy, On the type of certain $C_{0}$-semigroups, Comm. Part. Diff Eq., 18 (1993), 1299-1307.

[14] M. Renardy, On the linear stability of hiperbolic PDEs and viscoelastic flows, Z. Angew. Math. Phys., 45 (1994), 854-865.

[15] E. Pişkin, H. Yüksekkaya, Non-existence of solutions for a Timoshenko equations with weak dissipation, Mathematica Moravica, 22 (2018), 1-9.

[16] Q. P. Vua, J. M. Wang, G. Q. Xu, S. P. Yung, Spectral analysis and system of fundamental solutions for Timoshenko beams, Appl. Math. Lett., 18 (2005), 127134 .

\section{Ronaldo Ribeiro Alves}

Departament of Matematics

Federal University of SÃo João Del-Rey

36307-352, SÃo JoÃo DEL-REY, MG

BRAZIL

E-mail address: ronribal@ufsj.edu.br

\section{Jaime E. Muñoz Rivera}

Federal University of Rio de Janeiro and National Laboratory fOR Scientific Computation 25651-075, Petrópols, MG

BRAZIL

E-mail address: rivera@lncc.br

\section{Carlos A. Raposo}

Doctoral Program, Federal University of Bahia and Federal University of São JoÃo del-Rey 36307-352, SÃo Jõ̃o DEL-REY, MG

BRAZIL

E-mail address: raposo@ufsj.edu.br 